\title{
Performance of a fast, highly segmented electromagnetic calorimeter
}

\author{
W. Badgett, R. Ball, Y. Cha, J. Chapman, R.A. Dunn, R. Gustafson, M. Krasberg and R. Thun \\ University of Michigan, Ann Arbor, MI 48109, USA
}

Received 29 April 1991

We describe the energy, position, and angular resolutions of an electromagnetic calorimeter consisting of 56 layers of proportional wire and lead-filled tubes. The calorimeter was tested with two different electronic readout systems in a beam of electrons with energies ranging from 10 to $200 \mathrm{GeV}$.

\section{Introduction}

We present results from beam tests of a fast, finegrained electromagnetic calorimeter. This work was part of a program of research and development of detectors suitable for use at the Superconducting Supercollider (SSC). Our research was motivated specifically by a conceptual design of an SSC experiment dedicated to the measurement of muons produced in high-energy proton-proton collisions [1]. In such an experiment it is natural to consider the use of a thick absorber around the interaction point to filter muons from the intense flux of secondary hadrons. However, the correct interpretation of events containing muons may require knowledge of the other, coproduced particles, and one is led inevitably to consider some instrumentation of the absorber to measure the energy flow associated with these additional particles.

The use of proportional-wire technology for such absorber instrumentation has a number of attractions [2]. In particular, it is cheap and provides for the possibility of measuring simultaneously the location, direction, and energy of showering particles. The SSC environment is characterized by very high particle production rates and intense radiation levels [3]. Previous work [4] has shown that proportional wire chambers using small cells, fast gases, special pulse-shaping electronics, and operated at modest gas gains, can perform adequately in this environment.

To evaluate some of the concepts outlined above, we have constructed and tested a fine-grained electromagnetic calorimeter which could be attached to the frontend of a hadron absorber. Such a calorimeter could also be employed in any high-rate experiment that requires the precise location and direction of showering particles. The performance of the calorimeter was investigated with high-energy electrons provided by the Tagged
Photon Laboratory at Fermilab [5]. Measurements were made with two different electronic readout systems at beam energies of $10,25,50,100$, and $200 \mathrm{GeV}$. The main goal of the beam tests was to learn the dependence of energy and angular resolutions on gas gain and readout speed.

\section{Calorimeter design}

The electromagnetic calorimeter consists of 56 stacked planes of $0.472 \mathrm{~cm}$ wide square brass tubes. As shown in fig. 1, each plane contains 32 such tubes which are filled with lead or gas in an alternating sequence. Each gas-filled tube encloses a $38 \mu \mathrm{m}$ diameter gold-

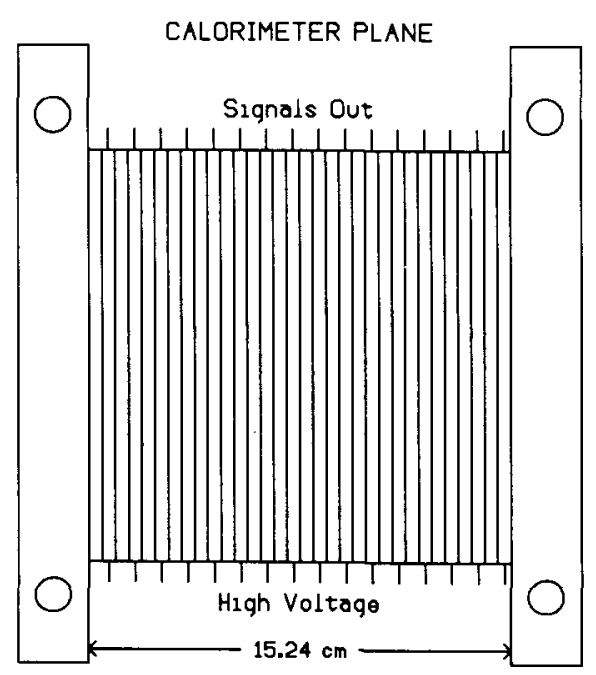

Fig. 1. Configuration of one of 56 identical calorimeter planes, consisting of an alternating sequence of lead-filled and proportional-wire tubes. 
Table 1

Geometry of the calorimeter

\begin{tabular}{|c|c|c|c|}
\hline \multicolumn{2}{|c|}{$Y$ orientation } & \multicolumn{2}{|c|}{$X$ orientation } \\
\hline 1 & 0 & 2 & 1 \\
\hline 3 & 1 & 4 & 0 \\
\hline 5 & 1 & 6 & 0 \\
\hline 7 & 0 & 8 & 1 \\
\hline 9 & 1 & 10 & 0 \\
\hline 11 & 0 & 12 & 1 \\
\hline 13 & 0 & 14 & 1 \\
\hline 15 & 1 & 16 & 0 \\
\hline 17 & 0 & 18 & 1 \\
\hline 19 & 1 & 20 & 0 \\
\hline 21 & 1 & 22 & 0 \\
\hline 23 & 0 & 24 & 1 \\
\hline 25 & 1 & 26 & 0 \\
\hline 27 & 0 & 28 & 1 \\
\hline 29 & 0 & 30 & 1 \\
\hline 31 & 1 & 32 & 0 \\
\hline 33 & 0 & 34 & 1 \\
\hline 35 & 1 & 36 & 0 \\
\hline 37 & 1 & 38 & 0 \\
\hline 39 & 0 & 40 & 1 \\
\hline 41 & 1 & 42 & 0 \\
\hline 43 & 0 & 44 & 1 \\
\hline 45 & 0 & 46 & 1 \\
\hline 47 & 1 & 48 & 0 \\
\hline 49 & 0 & 50 & 1 \\
\hline 51 & 1 & 52 & 0 \\
\hline 53 & 1 & 54 & 0 \\
\hline 55 & 0 & 56 & 1 \\
\hline
\end{tabular}

plated tungsten wire to which high voltage is applied through a $100 \mathrm{k} \Omega$ decoupling resistor. The sense wire is decoupled from the readout electronics by a $100 \mathrm{pF}$ capacitor. The planes are mounted on four steel rods in an alternating pattern of orthogonal measuring stations. To avoid open paths through the calorimeter, successive planes of a particular orientation ( $X$ or $Y$ ) have their first tube filled with either lead or gas as shown in table 1 , where offset $=0$ means a first tube filled with gas and offset $=1$ indicates one containing lead. This particular geometry, with planes of successive proportional and lead-filled tubes, was motivated by a desire to measure shower coordinates in every plane of a reasonably homogeneous calorimeter.

The active area of each plane has a cross section of $15.24 \times 15.24 \mathrm{~cm}$. Tubes within a plane are bonded to $0.25 \mathrm{~mm}$ thick G-10 fiberglass sheets, forming a strong sandwich structure. The planes are separated by an additional $0.15 \mathrm{~mm}$ thick mylar sheet when mounted on the steel support rods. The average separation between planes is $0.554 \mathrm{~cm}$, yielding a total calorimeter depth of $31.0 \mathrm{~cm}$. The interior cross section of the brass tubes is $0.396 \times 0.396 \mathrm{~cm}$. Each lead-filled tube was weighed to ensure the absence of voids. The radiation thickness of each layer, averaged over the surface, is equal to 0.40 producing an overall calorimeter depth of 22.4 radiation lengths.

We selected a mixture of $50 \%$ argon and $50 \%$ carbon dioxide as the proportional chamber gas since this produces very fast drift times in small cells, comparable to those achieved with CF4 mixtures [2]. The maximum drift time in the calorimeter cells is approximately 30 ns. The planes are connected in parallel to the gas distribution system via manifolds, and tubes within a plane are connected serially. Gas inside the calorimeter was kept at atmospheric pressure by operating with low total flow rates, typically $0.3 \mathrm{ft}^{3} / \mathrm{h}$, through a low-impedance exhaust. We made no corrections for variations in atmospheric pressure since most data runs were taken in sets over comparatively short intervals of time.

\section{Electronics}

The calorimeter was tested with two completely independent amplification and readout systems. The first of these consists of an already existing, CAMAC-compatible system [6] which amplifies the signal from individual tubes and stores the collected charge on an integrating capacitor. Approximately $700 \mathrm{~ns}$ after the initial trigger, this capacitor is decoupled from the input amplifier to preserve the charge for subsequent readout via a multiplexed 12-bit analog-to-digital converter (ADC). The system reads out only those channels with signals approximately 5 to 10 or more counts above pedestal. The noise per channel is less than 1 ADC count. Pedestals and gains are determined with calibration runs that inject test pulses into the amplifiers.

This electronic readout system has good noise characteristics and was used to establish the basic performance characteristics of the calorimeter. It is, however, too slow for operation in the high-rate environment of the SSC. For this reason we implemented a second readout system specifically aimed at fast signal processing. This system uses a tail-cancelling shaping amplifier [7] whose output signal is only $20 \mathrm{~ns}$ wide at its base. The gains of the amplifiers were adjusted to be uniform to within $0.5 \%$ (rms). The amplifier signal is digitized into a 12-bit word by a LeCroy 1885 ADC operated with an integrating gate width of $50 \mathrm{~ns}$, sufficient for the $30 \mathrm{~ns}$ maximum drift time. The ADC pedestals and gains are obtained from dedicated calibration runs. As described below, we also checked pedestals and noise by recording beam data at zero calorimeter operating voltage.

\section{Test beam}

The calorimeter was tested in the Proton East area of Fermilab with a beam consisting primarily of electrons. 
Data were obtained at beam energies of 10, 25, 50, 100 and $200 \mathrm{GeV}$. Electrons constituted approximately $90 \%$ of beam particles at energies of $100 \mathrm{GeV}$ or less. At 200 $\mathrm{GeV}$ this fraction dropped to about $80 \%$. The beam line was set to yield instantaneous rates of several hundred particles per second during the spill. The beam energy spread was not directly measured but was estimated to be less than $2 \%$ (rms). The calorimeter readout was triggered by the coincidence signal from small scintillation counters placed into the beam just in front of the calorimeter.

\section{Results}

Data were recorded at a number of beam energies in the range from 10 to $200 \mathrm{GeV}$. Following the history of the beam tests, we first present results obtained with the slow, low-noise electronic readout system. These data establish the basic calorimeter operating characteristics and are summarized in figs. 2-16. The test beam time was allocated according to a schedule which allowed only a limited set of measurements with the fast electronic system, and results from these measurements are presented in figs. 17-22.

The calorimeter signal, summed over all 896 channels, is shown in fig. 2 for beam energies of $25,50,100$, and $200 \mathrm{GeV}$ and for operating voltages of 1400,1600 ,

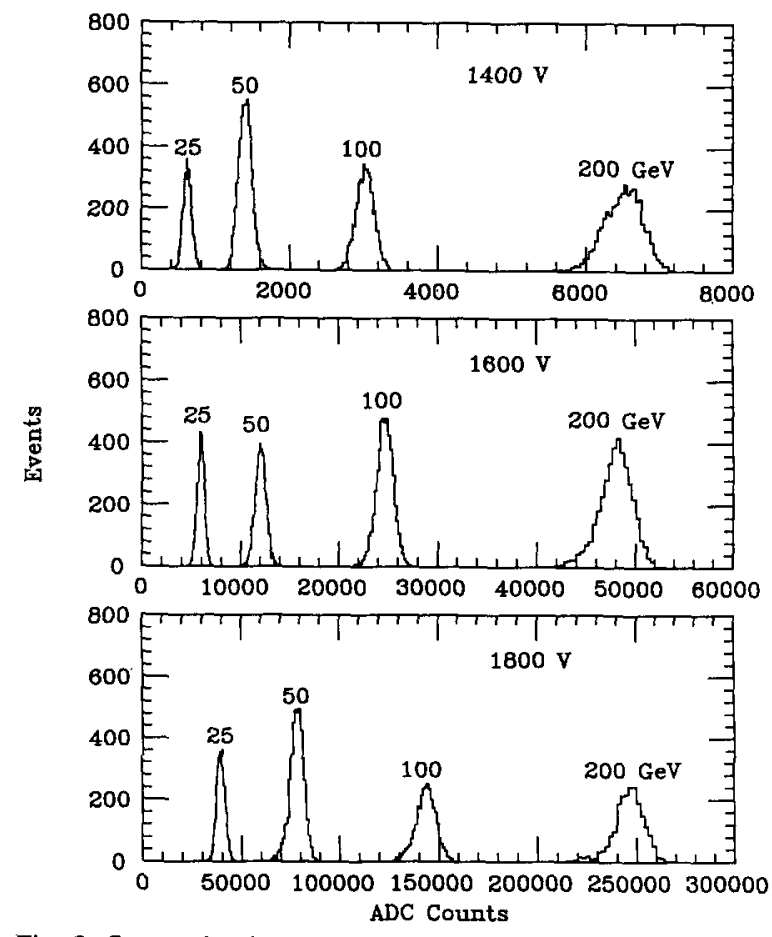

Fig. 2. Summed calorimeter signal for various beam energies and operating voltages.

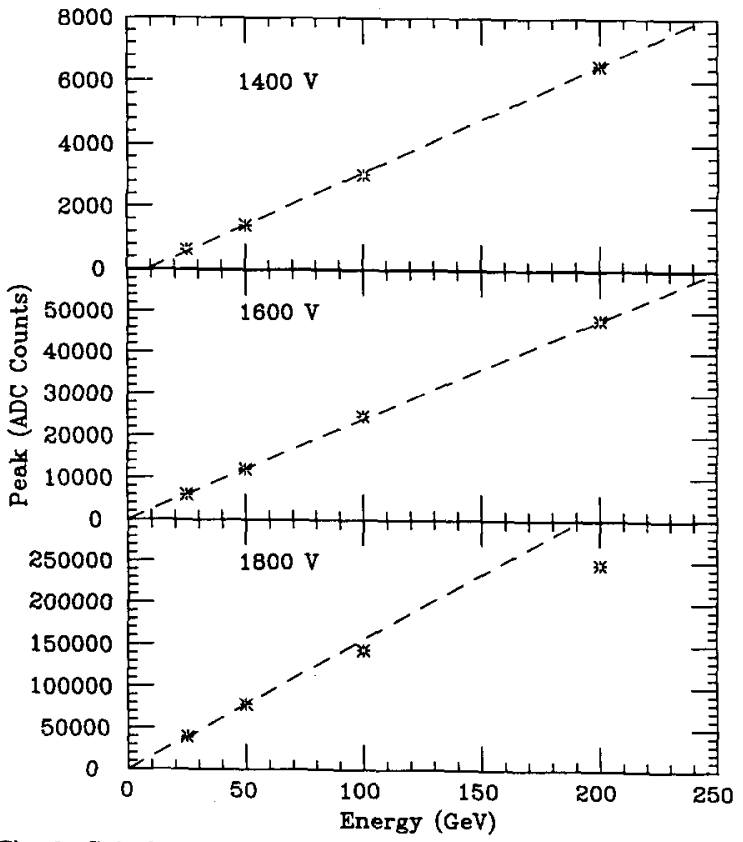

Fig. 3. Calorimeter response as a function of beam energy for three operating voltages.

and $1800 \mathrm{~V}$. The beam was well centered on the calorimeter when these data were recorded. The observed signal distributions were fitted with Gaussianshaped peaks whose means and widths were used to determine energy calibrations and resolutions. The mean values of the peaks are presented in fig. 3 as a function

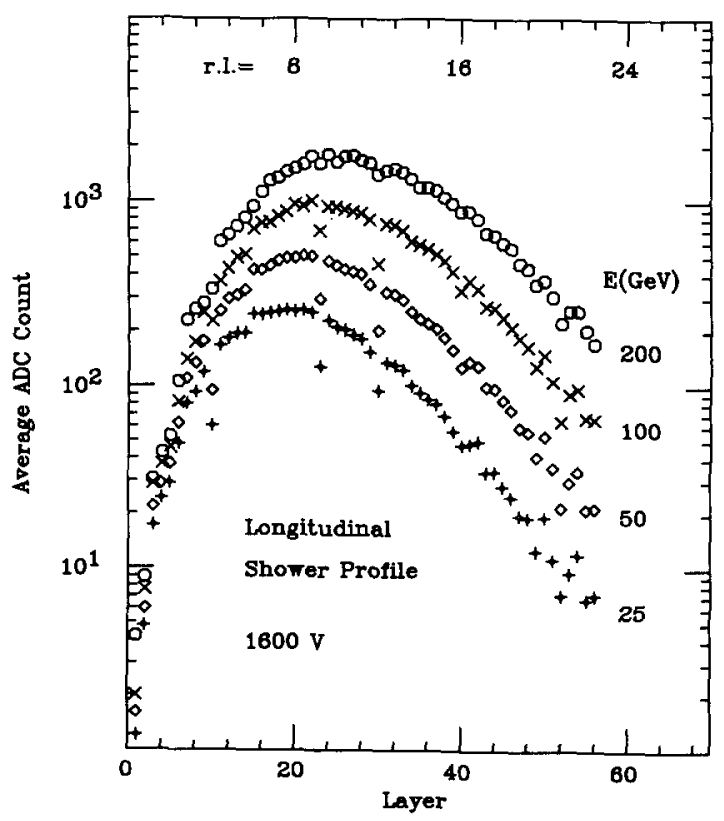

Fig. 4. Measured longitudinal shower profiles. 


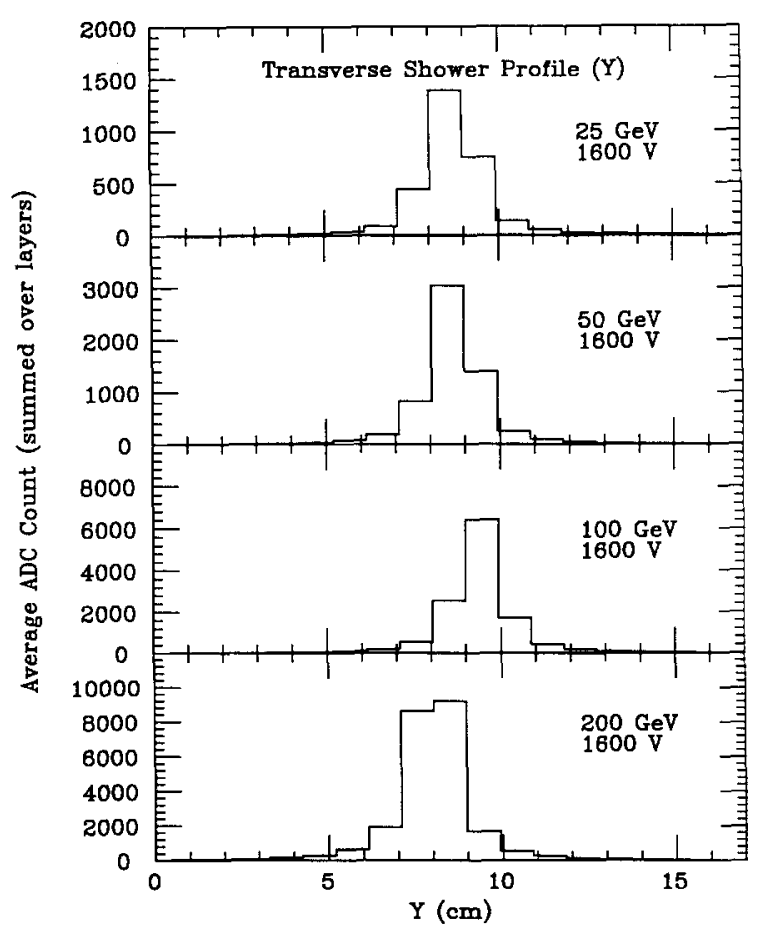

Fig. 5. Measured transverse shower profiles.

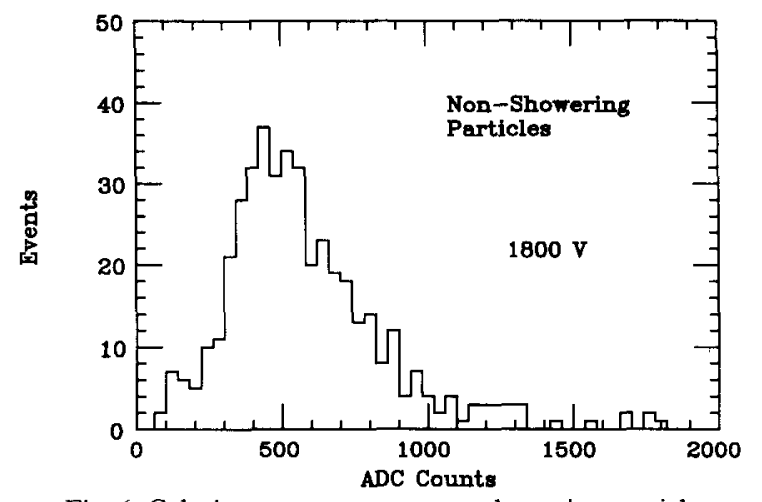

Fig. 6. Calorimeter response to nonshowering particles.

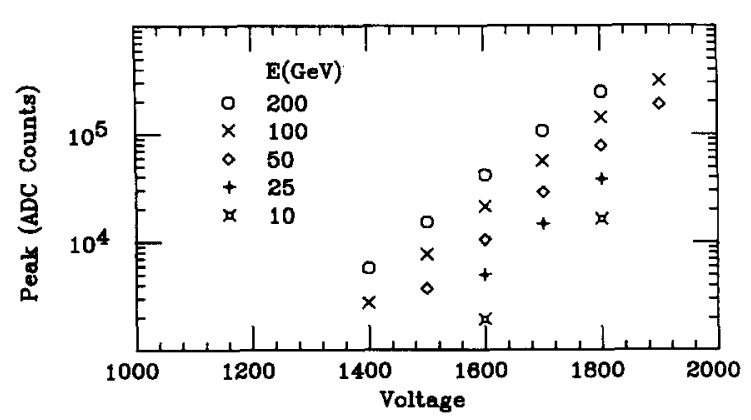

Fig. 7. Calorimeter response as a function of operating voltage.

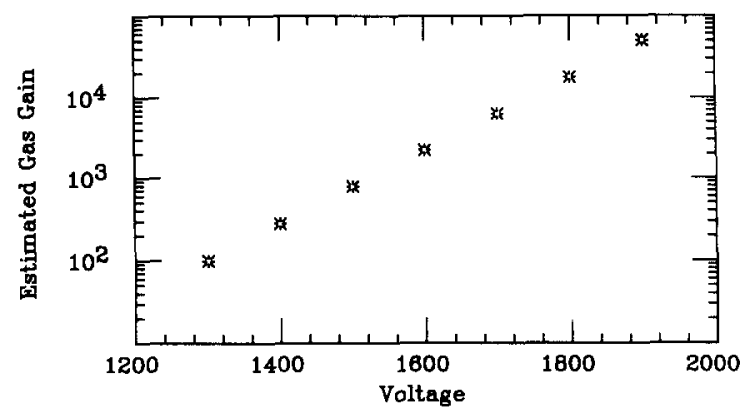

Fig. 8. Estimated calorimeter gas gain.

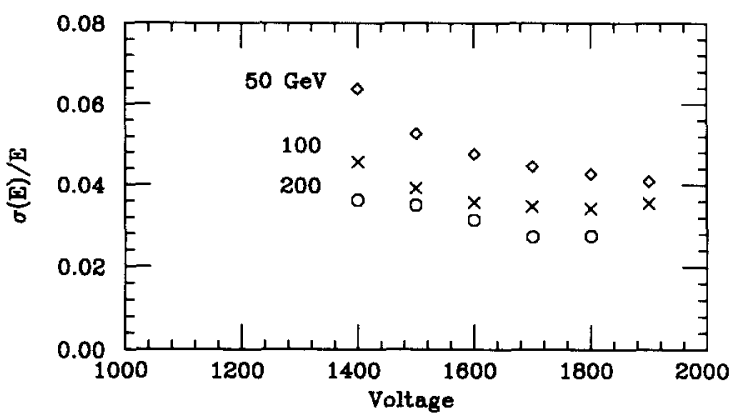

Fig. 9. Measured energy resolution as a function of operating voltage.

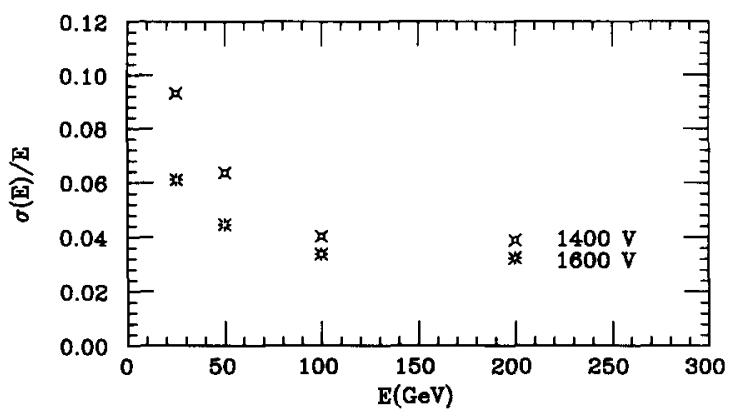

Fig. 10. Measured energy resolution as a function of beam energy.

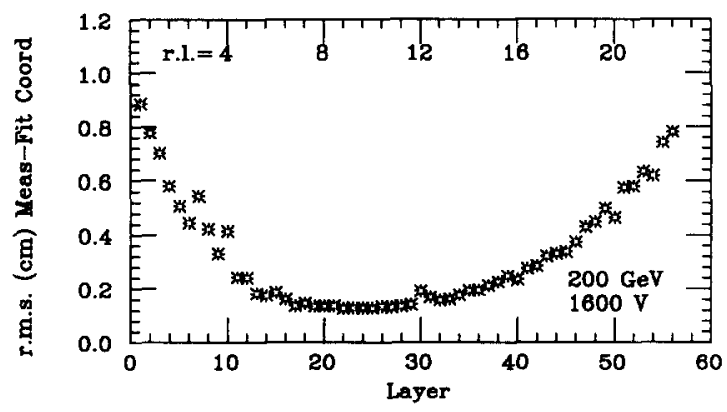

Fig. 11. Measured shower position resolution as a function of calorimeter layer. 


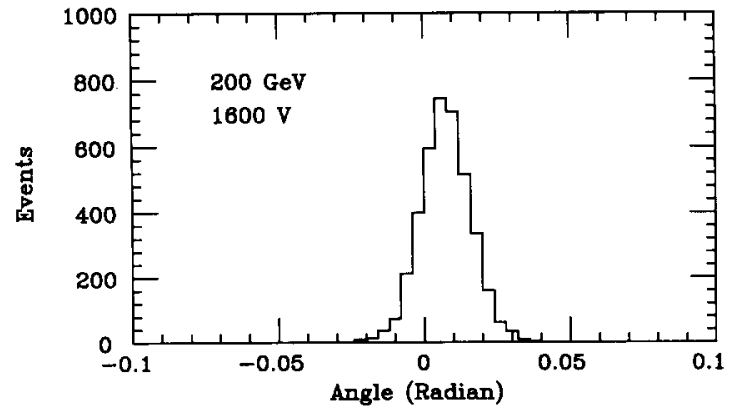

Fig. 12. Measured shower angular distribution in the horizontal $(X-Z)$ plane.

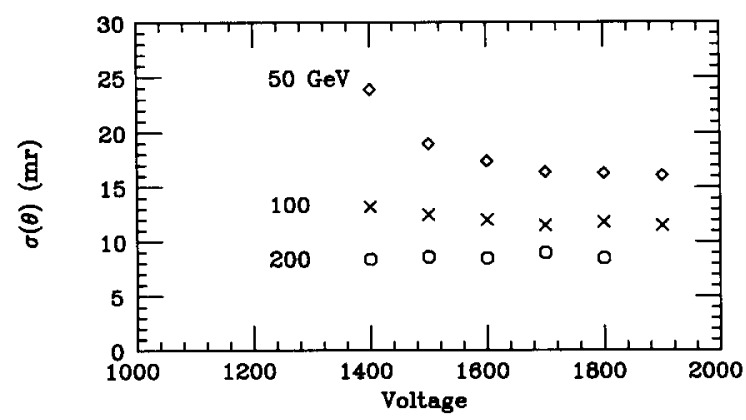

Fig. 13. Measured angular resolution as a function of operating voltage.

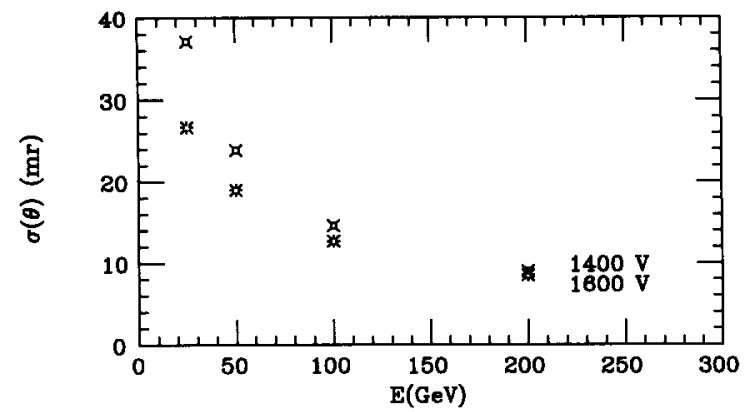

Fig. 14. Measured angular resolution as a function of beam energy.

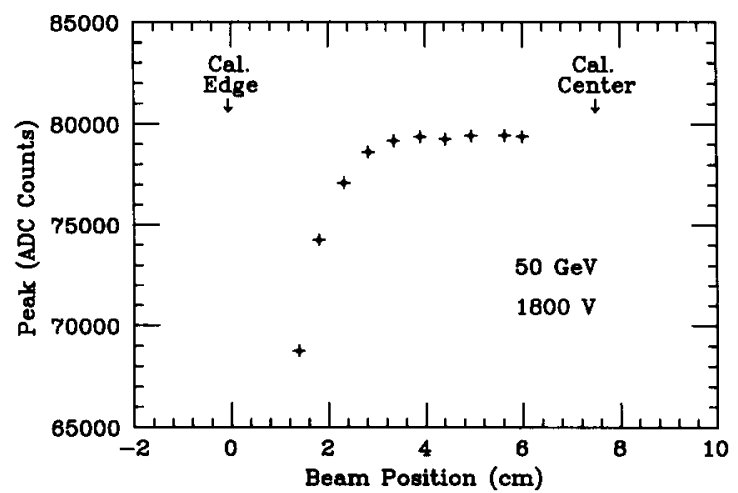

Fig. 15. Calorimeter response as a function of beam position.
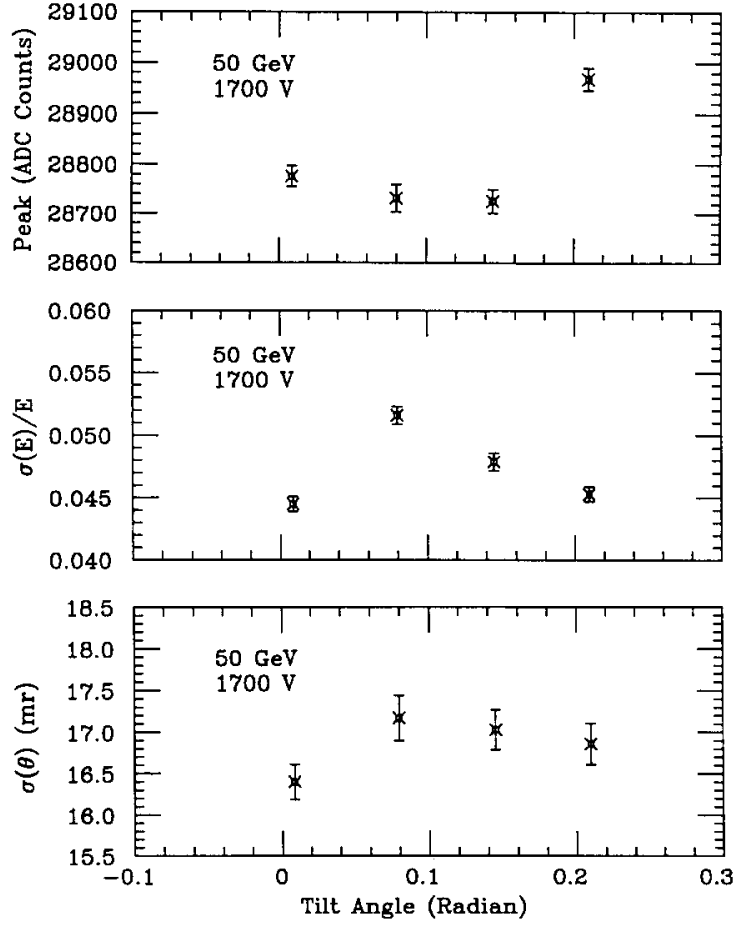

Fig. 16. Calorimeter response and resolutions as a function of beam angle of incidence (calorimeter tilt angle).

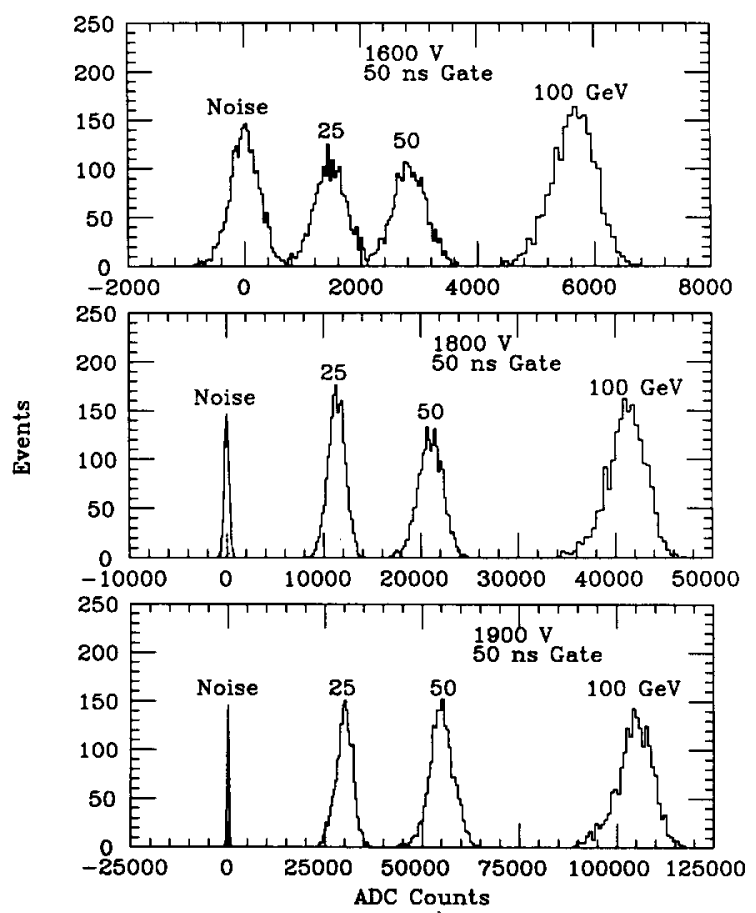

Fig. 17. Summed calorimeter signal for various beam energies and operating voltages (fast electronics). 


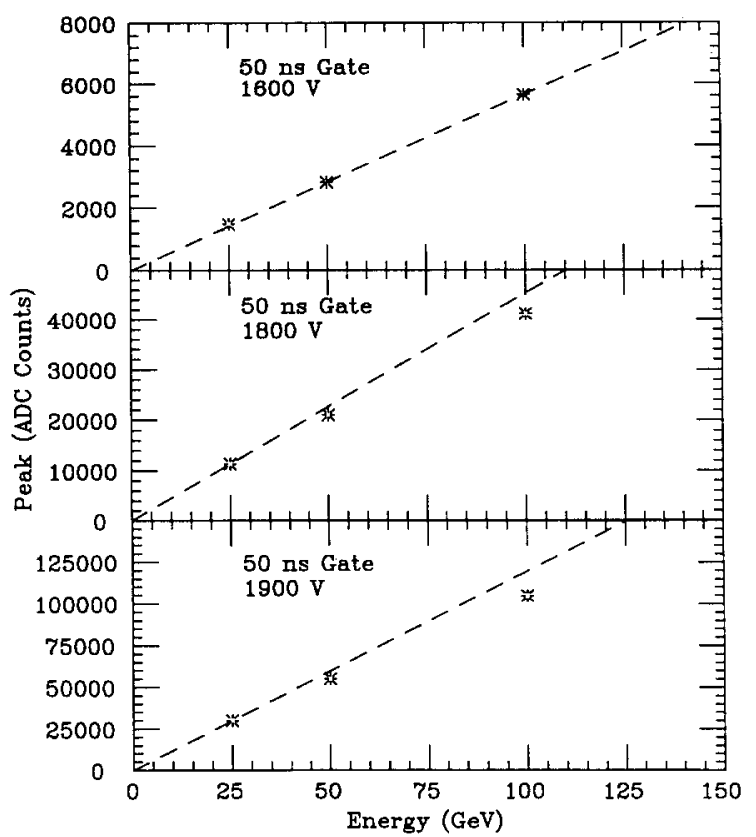

Fig. 18. Calorimeter response as a function of beam energy for three operating voltages (fast electronics).

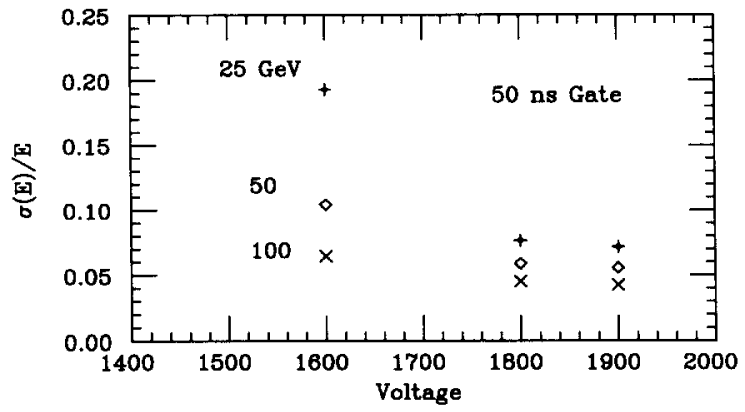

Fig. 19. Measured energy resolution as a function of operating voltage (fast electronics).

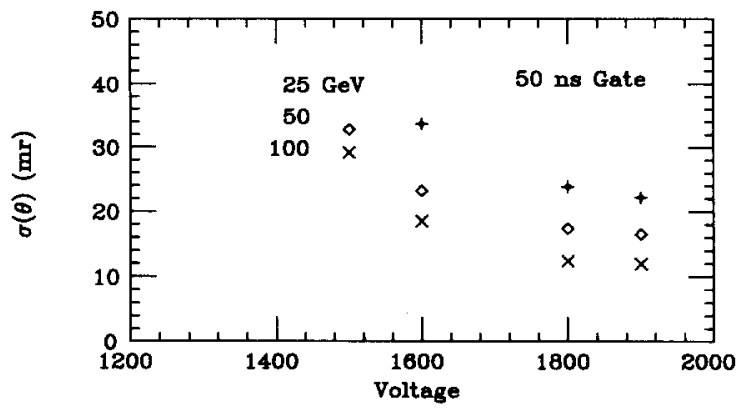

Fig. 20. Measured angular resolution as a function of operating voltage (fast electronics)

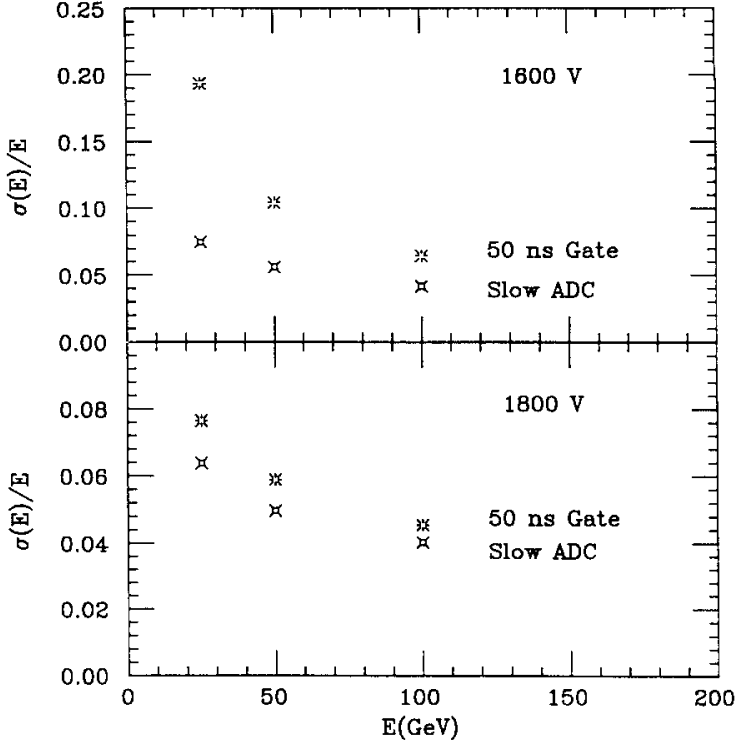

Fig. 21. Comparison of measured energy resolutions obtained with the slow and fast (" $50 \mathrm{~ns}$ Gate") electronics as a function of beam energy at two operating voltages.

of energy for the three different voltages. Several effects are observed. At $1400 \mathrm{~V}$ the extrapolated calorimeter response is negative at zero energy. This is an artifact of the sparse data scan which ignores cells with an ADC count less than 5 to 10 counts above pedestal. The impact of this truncation is significant only at low voltage where the fraction of cells with small signals is non-negligible. At $1600 \mathrm{~V}$ the behavior of the calorime-

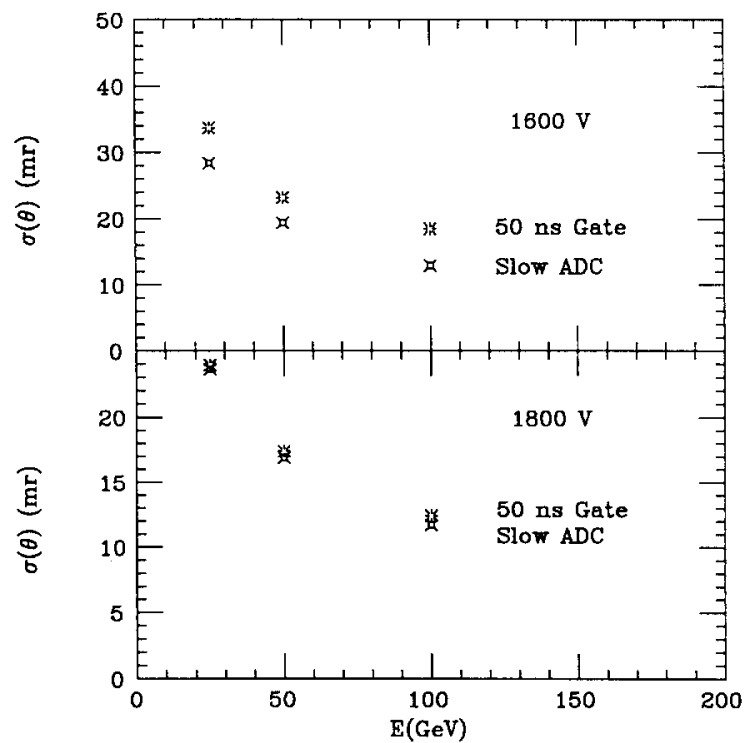

Fig. 22. Comparison of measured angular resolutions obtained with the slow and fast (" 50 ns Gate") electronics as a function of beam energy at two operating voltages. 
ter is very linear with a well behaved zero-energy intercept. Saturation effects are observed at $1800 \mathrm{~V}$. At 200 $\mathrm{GeV}$ a significant number of cells at the core of the shower exceed the ADC limit of 4095 counts. In addition, space-charge effects produce a visible non-linearity in the energy range from 0 to $100 \mathrm{GeV}$. Shower leakage is estimated to increase by about one percent between 100 and $200 \mathrm{GeV}$, and is not the dominant source of the observed nonlinearity.

The fine segmentation of the calorimeter allows a detailed measurement of shower shape as indicated in figs. 4 and 5. The reduced response in layers 10,23 , and 30 observed in the longitudinal shower profile is the result of several dead or inefficient cells in these layers. The shower shape at high energy is qualitatively in agreement with the predictions of ref. [8]. We note that, as indicated in fig. 5 , the transverse location of the beam varied slightly from run to run.

The gas gain of the calorimeter was estimated from the signal, shown in fig. 6 , produced by nonshowering beam particles such as muons and pions, and from the calorimeter response as a function of voltage presented in fig. 7. The gas gain varies approximately by a factor of 8.0 per $200 \mathrm{~V}$ change in operating voltage and is equal to about $2.2 \times 10^{3}$ at $1600 \mathrm{~V}$ as shown in fig. 8 .

The energy resolution of the calorimeter is displayed in figs. 9 and 10 as a function of voltage and beam energy. The observed resolution is parametrized reasonably well by the usual form

$\sigma(E) / E=\sqrt{a^{2} /(E / \mathrm{GeV})+(b)^{2}}$,

where the coefficient $a$ is 0.45 at $1400 \mathrm{~V}, 0.28$ at 1600 $\mathrm{V}$, and 0.24 at $1800 \mathrm{~V}$. The coefficient $b$ equals approximately 0.022 independent of voltage and originates from a combination of the unknown beam energy spread and possible calorimeter effects.

Shower directions were measured in both the $X-Z$ and $Y-Z$ planes where $Z$ defines the calorimeter axis and $X$ and $Y$ are the transverse coordinates. A shower coordinate ( $X$ or $Y$ ) was determined in each of the 56 calorimeter planes using a center-of-gravity (c.g.) algorithm, and these coordinates were fitted to a straight line by the least-square method. Substantially better angular resolutions were achieved by weighting the square of the deviation in each of the planes by the total ADC count in that plane. Weighting the fits by the actually observed deviations did not result in further, significant improvements. We therefore analyzed all data using the convenient ADC weighting method. Typically, a plane was included in the fits if it contained at least one cell with an ADC count of 10 or more. The observed angular resolutions were quite insensitive to this threshold or to the number of cells within a plane required to be above threshold.
Fig. 11 shows the rms positional resolution of each of the 56 layers at $200 \mathrm{GeV}$ and at an operating voltage of $1600 \mathrm{~V}$. The calculation of these rms values includes all c.g. coordinates within $\pm 2.5 \mathrm{~cm}$ of the shower axis. In the central layers the shower positional resolution is $0.14 \mathrm{~cm}$. It is substantially worse in the initial layers because the core of the shower does not produce a large signal there, especially if it strikes a lead-filled tube. Moreover, the narrow core of the front of the shower is surrounded by a relatively broad distribution of energy deposits from wide-angle and backscattered photons and electrons. The resolution is also worse towards the rear of the calorimeter as a result of the spatial spreading of the diminishing shower.

An example of a shower angular distribution (projected onto the $X-Z$ plane) is shown in fig. 12. The dependence of the angular resolution on beam energy and operating voltage is summarized in figs. 13 and 14 . The resolution improves with both energy and voltage and, like the energy resolution, has an approximate $1 / \sqrt{E}$ dependence. At $200 \mathrm{GeV}$ and $1800 \mathrm{~V}$ the projected shower axis is measured with a resolution of $\sigma(\theta)=8 \mathrm{mrad}$.

We also investigated the response of the calorimeter as a function of shower position and angle of incidence. Fig. 15 shows the peak value of the summed calorimeter signal as a function of transverse beam position. The response is very uniform until the beam approaches to within $3 \mathrm{~cm}$ of the calorimeter edge. At that point the signal begins to decrease smoothly as the beam is moved further towards the edge and shower leakage increases. We also recorded some data with the calorimeter tilted at several angles with respect to the beam. The behavior of the signal peak, energy resolution, and angular resolution as a function of tilt angle are displayed in fig. 16. Statistically significant effects are observed, but they do not represent large percentage variations in the measured quantities.

We now turn to the second phase of the measurements, those made with the pulse-shaping amplifiers and fast ADCs. The quality of these measurements was degraded by two factors which were not intrinsic to the basic setup but which we were not able to correct during our brief stay in the test beam. The more serious of these two problems was noise picked up by the $4.0 \mathrm{~m}$ long twisted-pair signal cables between the calorimeter and the fast shaping amplifiers. These long cables were inserted as a makeshift solution to an unexpected time delay in generating the ADC gate. A second, less serious problem was the discovery after measurements had been completed that the amplifiers for layers 15 and 16 were not functioning properly, and these layers were removed from the analysis. The basic impact of their removal was a decrease of the summed calorimeter signal by $6-9 \%$, depending on energy, and a reduction of the energy resolution by about $15 \%$. 
The calorimeter response with the fast electronics is shown in fig. 17 at beam energies of 25,50 , and 100 $\mathrm{GeV}$ and at operating voltages of 1600,1800 , and 1900 $V$. The noise peak was determined by recording data with the calorimeter voltage set to zero. It is clear that noise dominates the energy resolution at $1600 \mathrm{~V}$ and is an insignificant factor at $1900 \mathrm{~V}$ where the signal is much larger because of the increased gas gain. The mean values of the signal peaks are plotted in fig. 18 as a function of energy at the three operating voltages. At $1600 \mathrm{~V}$ one observes the same linear response seen in fig. 3 for the data taken with the slow electronics. The calibration of the fast ADCs was such that the 12 bits of the data word were not saturated at $100 \mathrm{GeV}$ even at $1900 \mathrm{~V}$, so that the observed nonlinearity at 1800 and $1900 \mathrm{~V}$ is the result of shower leakage and space-charge effects.

The measured energy and angular resolutions with the fast electronics are displayed in figs. 19 and 20. Again, resolutions improve with energy and operating voltage. We make a direct comparison in figs. 21 and 22 of calorimeter performance achieved with the two electronic readout systems. To obtain a fair comparison, layers 15 and 16 have been removed from the analysis of both sets of data. (As mentioned above, the amplifiers of layers 15 and 16 did not function properly in the fast-electronics setup labelled " $50 \mathrm{~ns}$ Gate" in the figures). At $1800 \mathrm{~V}$, where the noise described above and shown in fig. 17 has only a small effect, the energy resolution of the fast system is about $15 \%$ worse than that obtained with the slow electronics. We do not know the origin of this reduction in resolution but speculate that some loss of signal information occurs during the fast pulse-shaping process. There is essentially no difference in the angular resolutions observed at $1800 \mathrm{~V}$.

\section{Conclusions}

We have designed, constructed, and tested a finegrained electromagnetic calorimeter as part of the SSC Generic Detector R\& D Program. The calorimeter consists of 56 stacked planes of $0.472 \mathrm{~cm}$ wide square brass tubes filled with either proportional wires or lead and has a total depth of 22.4 radiation lengths. The proportional tubes were operated with a mixture of $50 \%$ argon and $50 \%$ carbon dioxide which yields a maximum drift time of about $30 \mathrm{~ns}$. The calorimeter was instrumented with two different electronic readout systems, one a previously existing, slow, low-noise setup, and the other based on pulse-shaping amplifiers and fast ADCs with a $50 \mathrm{~ns}$ integration gate. The calorimeter was tested at Fermilab with a beam of electrons at energies of 10,25 , 50,100 , and $200 \mathrm{GeV}$. Shower energy, position, and angular resolutions improved with energy and operating voltage. With the slow electronic system and the calorimeter operated at $1800 \mathrm{~V}$, we observe

$\sigma(E) / E=\sqrt{(0.24)^{2} /(E / \mathrm{GeV})+(0.022)^{2}}$,

where the constant term 0.022 arises from the unknown beam energy spread and possible calorimeter effects. The angular resolution at $1800 \mathrm{~V}$ is observed to be $\sigma(\theta)=0.114 / \sqrt{(E / \mathrm{GeV})} \mathrm{rad}$. Similar results are obtained with the fast electronic system at high energy and high operating voltage. The observed energy resolution is about $15 \%$ worse with the fast system, whereas the position and angular resolutions are essentially unchanged. At lower energies and operating voltages, the performance of the calorimeter with the fast electronic setup was significantly degraded by noise introduced by the long signal cables between the calorimeter and amplifiers. These long cables were a makeshift solution to an unforeseen timing problem. We believe that placement of the amplifiers on or very near the calorimeter would have greatly reduced this loss in performance for the case of small signals.

\section{Acknowledgements}

This work was supported by the SSC Laboratory with funds made available through the Generic Detector R\&D Program. We are grateful to J. Bensinger, T. Dombeck and $\mathrm{M}$. Gilchriese for their help in allocating resources and negotiating the use of the test beam at Fermilab. We greatly appreciate the assistance of $\mathrm{C}$. Hojvat, A. Malensek, A. Neubauer, and J. Volk for preparing test beam and experimental facilities at Fermilab for our use. J. Anderson, C. Moore, R. Pordes, and $\mathrm{V}$. White advised us in the implemention of the Fastbus readout code and we are grateful for their help. We acknowledge $\mathbf{J}$. Dicks of the Michigan Instrumentation Shop for fabricating most of the calorimeter components, R. Jacobs for assisting us in their assembly, and M. Sarraj for designing a number of electronic components and boards. Finally, we thank D. Nitz, B. Roe, and G. Snow for their advice and support during various phases of this research.

\section{References}

[1] R. Thun et al., Proc. Workshop on Experiments, Detectors, and Experimental Areas for the Supercollider, Berkeley (1987) p. 78.

[2] R. Thun, Proc. Summer Study on High Energy Physics in the 1990s, Snowmass (1988) p. 779.

[3] D. Groom, SSCL-SR-1054 (1990).

[4] L.G. Christophorou et al., Nucl. Instr. and Meth. 163 (1979) 141 ; 
J. Fischer et al., Nucl. Instr. and Meth. A238 (1985) 249;

R. Henderson et al., IEEE Trans. Nucl. Sci. NS-34 (1987) 528 ;

R. Thun, Nucl. Instr. and Meth. A273 (1988) 157.

[5] C. Halliwell et al., Nucl. Instr. and Meth. 102 (1972) 51.

[6] R. Ball et al., Nucl. Instr. and Meth. 197 (1982) 371.
[7] We used the Brookhaven designed shaping amplifier IO $532-01$, a similar version of which is described by J. Fischer et al. in ref. [4] above. We are grateful to V. Radeka for providing us with the circuit diagram and layout of this amplifier.

[8] D. Müller, Phys. Rev. D5 (1972) 2677. 